\section{Radar-Enabled Recovery of the Sutter's Mill Meteorite, a Carbonaceous Chondrite Regolith Breccia}

Peter Jenniskens, ${ }^{1,2 *}$ Marc D. Fries, ${ }^{3}$ Qing-Zhu Yin, ${ }^{4}$ Michael Zolensky ${ }^{5}$ Alexander N. Krot ${ }^{6}$ Scott A. Sandford, ${ }^{2}$ Derek Sears, ${ }^{2}$ Robert Beauford, ${ }^{7}$ Denton S. Ebel, ${ }^{8}{ }^{\prime}$ jon M. Friedrich, ${ }^{8,9}$ Kazuhide Nagashima, ${ }^{6}$ Josh Wimpenny, ${ }^{4}$ Akane Yamakawa, ${ }^{4}$ Kunihiko Nishiizumi, ${ }^{10}$ Yasunori Hamajima, ${ }^{11}$ Marc W. Caffee, ${ }^{12}$ Kees C. Welten, ${ }^{10}$ Matthias Laubenstein, ${ }^{13}$ Andrew M. Davis, ${ }^{14,15}$ Steven B. Simon, ${ }^{14}$ Philipp R. Heck, ${ }^{14,15}$ Edward D. Young, ${ }^{16}$ Issaku E. Kohl, ${ }^{17}$ Mark H. Thiemens, ${ }^{18}$ Morgan H. Nunn, ${ }^{18}$ Takashi Mikouchi, ${ }^{19}$ Kenji Hagiya, ${ }^{20}$ Kazumasa Ohsumi, ${ }^{21}$ Thomas A. Cahill, ${ }^{22}$ Jonathan A. Lawton, ${ }^{22}$ David Barnes, ${ }^{22}$ Andrew Steele, ${ }^{23}$ Pierre Rochette, ${ }^{24}$ Kenneth L. Verosub, ${ }^{4}$ ]érôme Gattacceca, ${ }^{24}$ George Cooper, ${ }^{2}$ Daniel P. Glavin, ${ }^{25}$ Aaron S. Burton, ${ }^{25,26}$ Jason P. Dworkin, ${ }^{25}$ Jamie E. Elsila, ${ }^{25}$ Sandra Pizzarello, ${ }^{27}$ Ryan Ogliore, ${ }^{6}$ Phillipe Schmitt-Kopplin, ${ }^{28,29}$ Mourad Harir, ${ }^{28}$ Norbert Hertkorn, ${ }^{28}$ Alexander Verchovsky, ${ }^{30}$ Monica Grady, ${ }^{30}$ Keisuke Nagao, ${ }^{31}$ Ryuji Okazaki, ${ }^{32}$ Hiroyuki Takechi, ${ }^{32}$ Takahiro Hiroi, ${ }^{33}$ Ken Smith, ${ }^{34}$ Elizabeth A. Silber, ${ }^{35}$ Peter G. Brown, ${ }^{35}$ jim Albers, $^{1}$ Doug Klotz, ${ }^{36}$ Mike Hankey, Robert Matson, ${ }^{38}$ jeffrey A. Fries, ${ }^{39}$ Richard J. Walker, ${ }^{40}$ Igor Puchtel, ${ }^{40}$ Cin-Ty A. Lee, ${ }^{41}$ Monica E. Erdman, ${ }^{41}$ Gary R. Eppich, ${ }^{42}$ Sarah Roeske, ${ }^{4}$ Zelimir Gabelica, ${ }^{43}$ Michael Lerche, ${ }^{44}$ Michel Nuevo, ${ }^{1,2}$ Beverly Girten, ${ }^{2}$ Simon P. Worden ${ }^{2}$ (the Sutter's Mill Meteorite Consortium)

Doppler weather radar imaging enabled the rapid recovery of the Sutter's Mill meteorite after a rare 4-kiloton of TNT-equivalent asteroid impact over the foothills of the Sierra Nevada in northern California. The recovered meteorites survived a record high-speed entry of 28.6 kilometers per second from an orbit close to that of Jupiter-family comets (Tisserand's parameter $=2.8 \pm 0.3$ ). Sutter's Mill is a regolith breccia composed of CM (Mighei)-type carbonaceous chondrite and highly reduced xenolithic materials. It exhibits considerable diversity of mineralogy, petrography, and isotope and organic chemistry, resulting from a complex formation history of the parent body surface. That diversity is quickly masked by alteration once in the terrestrial environment but will need to be considered when samples returned by missions to C-class asteroids are interpreted.

$\mathrm{O}$ 22 April 2012, the KBBX (Beale Air Force Base, California), KDAX (Sacramento, California), and KRGX (Reno, Nevada) weather radars of the U.S. National Climatic Data Center's NEXRAD network (1) detected radial Doppler shifts in four sweeps, following a fast-moving daytime fireball seen over much of California and Nevada at 14:51:12 to 17 UTC (Fig. 1). The falling meteorites were

${ }^{1}$ SETI Institute, 189 Bernardo Avenue, Mountain View, CA 94043, USA. ${ }^{2}$ NASA Ames Research Center, Moffett Field, CA 94035, USA. ${ }^{3}$ Planetary Science Institute, Tucson, AZ 85719 2395, USA. ${ }^{4}$ Department of Geology, University of California at Davis, Davis, CA 95616, USA. ${ }^{5}$ Astromaterials Research and Exploration Science, NASA Johnson Space Center, Houston, TX 77058, USA. ${ }^{6}$ Hawai'i Institute of Geophysics and Planetology and Astrobiology Institute, University of Hawai'i at Mānoa, Honolulu, HI 96822, USA. ${ }^{7}$ Arkansas Center for Space and Planetary Sciences, University of Arkansas, AR 72701, USA. ${ }^{8}$ Department of Earth and Planetary Sciences, American Museum of Natural History, New York, NY 10024, USA. 'Department of Chemistry, Fordham University, Bronx, NY 10458, USA. ${ }^{10}$ Space Sciences Laboratory, University of California, Berkeley, CA 94720-7450, USA. ${ }^{11}$ Low Level Radioactivity Laboratory, Kanazawa University, Nomi, Ishikawa 923-1224, Japan. ${ }^{12}$ Department of Physics, Purdue University, West Lafayette, IN 47907, USA. ${ }^{13}$ Istituto Nazionale di Fisica Nucleare, Laboratori Nazionali del Gran Sasso, I-67100 Assergi, Italy. ${ }^{14}$ Department of the Geophysical Sciences, Enrico Fermi Institute and Chicago Center for Cosmochemistry, The University of Chicago, Chicago, IL 60637, USA. ${ }^{15}$ Robert A. Pritzker Center for Meteoritics and the area (2). One meteorite fell at Sutter's Mill (SM), the gold discovery site that initiated the California Gold Rush. Two months after the fall, SM find numbers were assigned to the 77 meteorites listed in table S3 (3), with a total mass of $943 \mathrm{~g}$. The biggest meteorite is $205 \mathrm{~g}$.

This is a tiny fraction of the pre-atmospheric mass, based on the kinetic energy derived from infrasound records. Eyewitnesses reported hearing a loud boom followed by a deep rumble. Infrasound signals (table S2A) at stations I57US and I56US of the International Monitoring System (4), located $\sim 770$ and $\sim 1080 \mathrm{~km}$ from the source, are consistent with stratospherically ducted arrivals (5). The combined average periods of all phase-aligned stacked waveforms at each station of $7.6 \mathrm{~s}$ correspond to a mean source energy of $4.0(-2.2 /+3.4) \mathrm{kT}$ of TNT, using the multistation period yield relation from (5). This was the most energetic reported bolide falling on land globally (6) since the 1.2-kT impact of asteroid $2008 \mathrm{TC}_{3}$ over Sudan in 2008 (7).

Seismic data suggest a point source altitude of $54.8 \pm 10.9 \mathrm{~km}$ above mean sea level, estimated from impulsive phase arrivals of the air blast at eight seismograph stations ( 8 ) by applying a simple half-space sonic velocity model and direct ray paths to a standard earthquake location code (table S2B).

This altitude corresponds to a persistent flare detected in a set of three photographs from Rancho Haven, north of Reno, Nevada (fig. S1). Triangulation with videos from Johnsondale, California, and Incline Village, Nevada (figs. S2 and S3), shows that the bolide was first detected at $90 \mathrm{~km}$ approaching from the east, had a broad peak in brightness around $56 \mathrm{~km}$, and detonated at $47.6 \pm$ $0.7 \mathrm{~km}$ (Table 1). Even in the daytime sky, a great many fragments were detected down to $30 \mathrm{~km}$.

The entry speed is twice that of typical triangulated falls from which meteorites have been recovered (table S1). SM has the highest disruption altitude on record. With an entry velocity of $28.6 \mathrm{~km} / \mathrm{s}$, the infrasound-derived kinetic energy corresponds to a pre-atmospheric mass of $\sim 40,000$

6AA, UK. ${ }^{31}$ Geochemical Research Center, University of Tokyo, Bunkyo-ku, Tokyo 113-0033, Japan. ${ }^{32}$ Department of Earth and Planetary Sciences, Kyushu University, Hakozaki, Fukuoka 812-8581, Japan. ${ }^{33}$ Department of Geological Sciences, Brown University, Providence, RI 02912, USA. ${ }^{34}$ Nevada Seismological Laboratory, University of Nevada, Reno, NV 89557, USA. ${ }^{35}$ University of Western Ontario, London, Ontario N6A 3K7, Canada. ${ }^{36}$ Space Science for Schools, Incline Village, NV 89451, USA. ${ }^{37}$ American Meteor Society, Geneseo, NY 14454, USA. ${ }^{38}$ Science Applications International Corporation, Seal Beach, CA 90740, USA. ${ }^{39}$ U.S. Air Force Weather Agency, 1st Weather Group, Offutt Air Force Base, NE 68113, USA. ${ }^{40}$ Department of Geology, University of Maryland, College Park, MD 20742, USA. ${ }^{41}$ Department of Earth Science, Rice University, Houston, TX 77005, USA. ${ }^{42}$ Glenn Seaborg Institute, Lawrence Livermore National Laboratory, Livermore, CA 94550, USA. ${ }^{43}$ Université de Haute Alsace, F-68093 Mulhouse, France. ${ }^{44}$ McClellan Nuclear Research Center, University of California at Davis, McClellan, CA 95652, USA.

${ }^{*}$ To whom correspondence should be addressed. E-mail: petrus.m.jenniskens@nasa.gov 
(range 20,000 to 80,000$) \mathrm{kg}$. Counter to intuition, the catastrophic disruption was key to meteorite survival from this fast entry (7). So far, $\sim 0.1 \mathrm{~kg} / \mathrm{km}$ estimated total fallen mass $\geq 1.7 \mathrm{~kg}$. This is far less than that recovered from the similar-sized but slower-impacting $2008 \mathrm{TC}_{3}: 1.2 \mathrm{~kg} / \mathrm{km}$ and $39 \mathrm{~kg}$ (7). An airship search did not find impact scars from falling kilogram-sized meteorites.

The pre-atmospheric orbit (Table 1) had low inclination and high eccentricity, with aphelion close to the orbit of Jupiter and perihelion $(q)$ approaching the orbit of Mercury (fig. S5). Semimajor axis, eccentricity, and inclination are strikingly similar to the preliminary values reported for the CM-type carbonaceous chondrite Maribo (9) by Haack et al. (10). The entry conditions, too, were much alike. Upon request, W. Singer and G. Stober provided the seven Juliusruh radar head-echo range and direction positions of the early 112- to $79-\mathrm{km}$ altitude part of this fall (table S1), which translated into the orbital elements listed in Table 1. SM and Maribo have lower perihelion distance and highereccentricity orbits than all other known (mostly ordinary chondrite) falls (table S1).

That distinction may be due to a bias toward a more recent evolution into Earth-crossing orbits for this population. SM has a cosmic ray exposure (CRE) age at the extreme low end of the CM2 chonhas been recovered along the trend line, for an

drite CRE age distribution, which as a group is younger than all other classes of meteorites except lunar meteorites (11). An age of $0.10 \pm 0.04$ million years (My) was obtained from the measured ${ }^{26} \mathrm{Al}$ activity (with a 0.705 million-year half-life) of $3.8 \pm 0.8 \mathrm{dpm} / \mathrm{kg}$ in SM36 (table S20). He and Ar isotopic ratios in SM43 and SM51 show no clear excess of a cosmogenic noble gas component, but a small excess of cosmogenic Ne is observed as deviations from two mixing lines between the trapped components; solar $\mathrm{Ne}-\mathrm{P} 3 \mathrm{Ne}$ and P3 Ne - Ne E $(12,13)$ (fig. S27). The low concentration of cosmogenic ${ }^{21} \mathrm{Ne}\left[1.02 \pm 0.11 \times 10^{-10} \mathrm{~cm}^{3} / \mathrm{g}\right.$ at standard temperature and pressure (STP)] indicates a very short CRE age of $0.051 \pm 0.006 \mathrm{My}$ (the average of four ages in table S18), if a production rate of $2 \times 10^{-9} \mathrm{~cm}^{3} / \mathrm{g} /$ My at STP for the near surface of a large object is adopted (14).

The SM and Maribo orbits have a Tisserand's parameter with respect to Jupiter $\left(T_{\mathrm{J}}\right)$ that borders those of asteroids $\left(T_{\mathrm{J}}>3\right)$ and Jupiter family comets $\left(T_{\mathrm{J}}=2\right.$ to 3$)$. Visual observations of Murchison $(\mathrm{CM})$ also point to approach on a low-inclined orbit (15). A possible Jupiter-family comet origin is intriguing (10) [for a review, see (15)], especially because the CM-like micrometeorites are dynamically linked to Jupiter-family comets (10). Finding two asteroids on orbits at $q=0.47$ astronomical unit $(\mathrm{AU})$ could mean that both are part

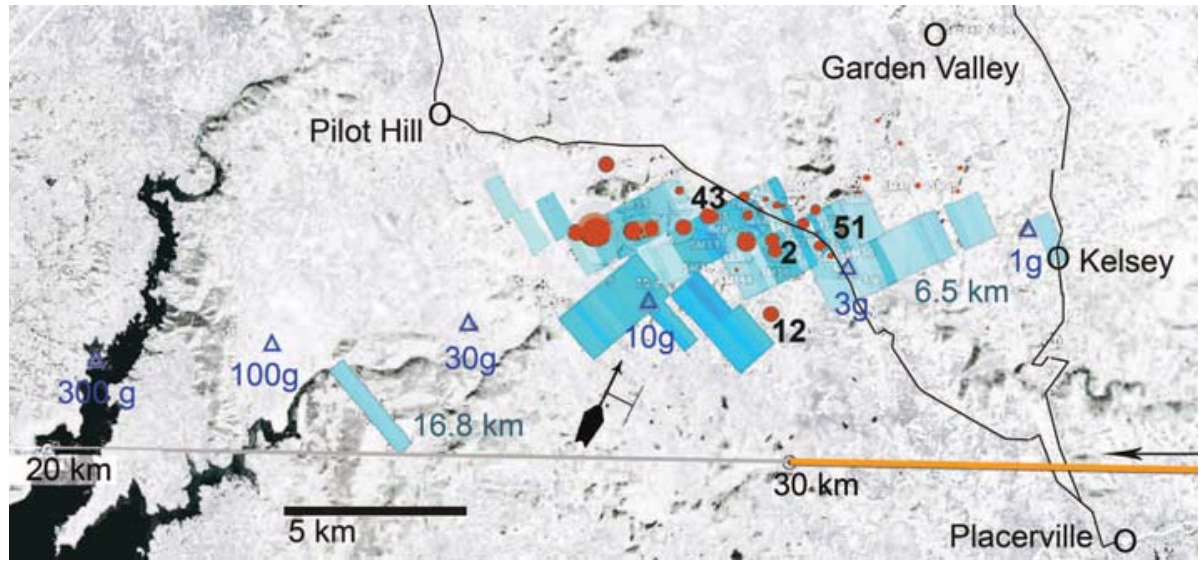

Fig. 1. Map of ground-projected fireball trajectory solution (orange line), radar Doppler reflectivity (light blue; $-5 \mathrm{dBZ}$ for pale blue to $+15 \mathrm{dBZ}$ for deepest blue), and meteorite find locations near Sutter's Mill (red dots are of size proportional to mass; relevant finds are labeled with SM number). Blue triangles are the calculated impact locations for breakup at $48 \mathrm{~km}$ and wind drift from $13 \mathrm{~m} / \mathrm{s}$ winds to azimuth $27^{\circ}$ between 18 and $2 \mathrm{~km}$ altitude. of an old $\sim 0.1$-My-old meteoroid stream, perhaps related to $2 \mathrm{P} /$ Encke (10), but only if CM chondrites survive longer than typical Taurid meteoroids.

Until other evidence of hydrothermal alteration in Jupiter-family comets is found (15), an origin in the asteroid belt is more likely. The asteroidfamily source has low inclination and is close to the 3:1 mean motion resonance with Jupiter, the CRE age leaving little time for thermal drag forces to move the semimajor axis of a main-belt asteroid into resonance at $2.5 \mathrm{AU}$. A suitable candidate is the (495) Eulalia family, recently proposed as a source of near-Earth C-class asteroids (17). Unlike ordinary chondrites ejected from the 3:1 resonance, which tend to collide with Earth on higher-impactprobability perihelion distance $q \sim 1$ AU orbits of lower eccentricity (table $\mathrm{S} 1$ ), the fractured $\mathrm{CM}$ chondrites may disintegrate too rapidly to evolve into such orbits. The short exposure age of SM as compared to other CM chondrites could mean it had already broken from a larger precursor while evolving into an SM-like orbit.

The reflectance spectrum of SM12 (fig. S8) is a good match to the Hayabusa 2 mission's target, asteroid $1999 \mathrm{JU}_{3}$, over the measured 0.38 to $0.92-\mu \mathrm{m}$ range when normalized at $0.55 \mu \mathrm{m}$ (fig. S8). The meteorite's albedo at $0.55 \mu \mathrm{m}$ is low, 2.5 to $4.0 \%$ at the standard $30^{\circ}$ incidence and $0^{\circ}$ emergence angles. Carbonates are abundant in this sample and produce strong infrared absorption bands near 1450 and $875 \mathrm{~cm}^{-1}$, stronger than those seen in CM2 Murchison.

SM12 has a compression strength of $82 \pm 6$ $\mathrm{MPa}$, as compared to $\sim 30 \mathrm{MPa}$ for other CM2s (18). The impacting asteroid fragmented at a dynamic pressure of only $0.9 \mathrm{MPa}$ at $48 \mathrm{~km}$, presumably because of internal cracks (19). X-ray computed tomography scanning (20) of SM3, 9, 18, 51, 54, and 73 at the 12 - to $30-\mu \mathrm{m} /$ voxel edge showed abundant fractures through stones and abundant fractures within some lithic fragments, terminating at the fragment edges (fig. S28). Volume measurements (table S19) yielded densities of $2.27 \pm 0.07 \mathrm{~g} / \mathrm{cm}^{3}$, similar to an average CM2 density of $2.20 \mathrm{~g} / \mathrm{cm}^{3}$ (range 1.88 to 2.47) (21). He ideal-gas pycnometry of SM19 gave a bulk density of $2.31 \pm 0.04 \mathrm{~g} / \mathrm{cm}^{3}$, a grain density of $3.34 \pm 0.02 \mathrm{~g} / \mathrm{cm}^{3}$, and a high porosity of $31.0 \pm 1.4 \%(22)$.

This density and the infrasound-derived kinetic energy yield an asteroid diameter of 2.5 to

Table 1. Atmospheric trajectory and pre-atmospheric orbit for the SM and Maribo CM chondrites. Angular elements are for equinox J2000.0.

\begin{tabular}{|c|c|c|c|c|c|}
\hline Atmospheric trajectory & SM & Maribo & Pre-atmospheric orbit & SM & Maribo \\
\hline$H_{\mathrm{b}}$ (beginning height $-\mathrm{km}$ ) & $90.2 \pm 0.4$ & $111.8 \pm 0.4$ & $T_{\mathrm{J}}$ (Tisserand's parameter) & $2.81 \pm 0.32$ & $3.04 \pm 0.32$ \\
\hline$H_{\mathrm{m}}($ broad maximum $-\mathrm{km})$ & $\sim 56$ & $\sim 58$ & $a$ (semimajor axis - AU) & $2.59 \pm 0.35$ & $2.34 \pm 0.29$ \\
\hline$H_{\mathrm{f}}($ disruption $-\mathrm{km})$ & $47.6 \pm 0.7$ & $37.3 \pm 0.6$ & $e$ (eccentricity) & $0.824 \pm 0.020$ & $0.795 \pm 0.026$ \\
\hline$V_{\infty}($ entry speed $-\mathrm{km} / \mathrm{s})$ & $28.6 \pm 0.6$ & $28.0 \pm 0.7$ & $\omega$ (argument of perihelion $-^{\circ}$ ) & $77.8 \pm 3.2$ & $99.0 \pm 1.4$ \\
\hline$h$ (entry elevation angle - ${ }^{\circ}$ ) & $26.3 \pm 0.5$ & $30.2 \pm 0.5$ & $\Omega$ (longitude of ascending node $-{ }^{\circ}$ ) & $32.77 \pm 0.06$ & $117.64 \pm 0.05$ \\
\hline$a_{\mathrm{z}}$ (entry azimuth angle from south $-^{\circ}$ ) & $272.5 \pm 0.4$ & $276.2 \pm 0.2$ & $i$ (inclination $-^{\circ}$ ) & $2.38 \pm 1.16$ & $0.72 \pm 0.98$ \\
\hline $\mathrm{Dec}_{\mathrm{g}}$ (geocentric declination of radiant $-{ }^{\circ}$ ) & $12.7 \pm 1.7$ & $18.8 \pm 1.6$ & Epoch (UT) & $2012-04-22.620$ & 2009-01-17.798 \\
\hline
\end{tabular}


$4.0 \mathrm{~m}$. Concentrations of the cosmogenic radionuclide ${ }^{60} \mathrm{Co}$ in three fragments-SM18, SM36 (table S20A), and SM43 - and model calculations (23) confirm that the asteroid's pre-atmospheric size was $>0.9 \mathrm{~m}$. The fireball's peak luminosity ( -18 to -20 magnitude) at the first broad maximum suggests a size of 1.8 to $3.5 \mathrm{~m}$.

Textural and compositional variety. Because of its large size, SM provides insight into the variety of materials present at the surface of its parent body. Individual SM meteorites have differing magnetic susceptibilities $\left(\chi\right.$ in $10^{-9}$ $\mathrm{m}^{3} / \mathrm{kg}$ ). Ten different stones (table S4) suggest a bimodal distribution clustering around $\log _{10} \chi=$ 4.03 and 4.26 . The primary magnetic mineral is magnetite $\left(\mathrm{Fe}_{3} \mathrm{O}_{4}\right)$, with concentrations of 2.0 and 3.3 weight $\%$ (wt \%), respectively. These magnetite concentrations are intermediate between typical CM2s $(\sim 1 \mathrm{wt} \%)$ and magnetite-rich $\mathrm{C} 2 \mathrm{~s}$ or anomalous $\mathrm{CMs}(>6 \mathrm{wt} \%)(24)$. SM2 has a stable natural remnant magnetization, probably extraterrestrial in origin, corresponding to a magnetic field paleointensity of $\sim 3 \mu \mathrm{T}$, comparable to other carbonaceous chondrites (25).

$\mathrm{SM}$ is a regolith breccia. Like all CM chondrites (26), SM contains Ne from solar wind implantation in a surface regolith (fig. S27). Unlike most other $\mathrm{CM}$ chondrites, the brecciated nature of that regolith is evident: SM2, 18, 47, 48, 51, and 54 contain angular to rounded clasts embedded in a fine-grained comminuted matrix seen visually (Fig. 2A), by $\mathrm{x}$-ray and backscattered electron mapping (Fig. 2B and fig. S13), and by x-ray and neutron computed tomography (figs. S28 and S30).

The classification of SM as a CM chondrite is confirmed by whole-rock chemistry (Fig. 3) and by $\mathrm{O}$ (Fig. 4 and fig. S21), Os (supplementary text $\mathrm{S} 2.6$ ), and $\mathrm{Cr}$ (supplementary text S2.7) isotopic compositions. The Os isotopic compositions and highly siderophile element abundances are well within the range of CM chondrites (figs. S16 and $\mathrm{S} 17)$. However, the Re-Os isotopic systematics indicate minor, what was probably recent, opensystem behavior of these two elements, as seen in other chondrites (27). SM plots in the field of CM chondrites (28) on the diagram of $\varepsilon^{54} \mathrm{Cr}$ versus $\Delta^{17} \mathrm{O}$ (fig. S20). Using ${ }^{53} \mathrm{Mn}-{ }^{53} \mathrm{Cr}$ chronometry $\left({ }^{53} \mathrm{Mn}\right.$ decays to ${ }^{53} \mathrm{Cr}$ with a half-life of $3.7 \mathrm{My}$ ), the data place the accretion time of SM at $4566.57 \pm 0.66$ million years ago (Ma) (fig. S19) (28).

SM contains CM lithologies (from CM2.0 to 2.1, Fig. 2B) with varying histories of aqueous alteration and thermal metamorphism. The abundances of thermally labile elements, such as Se, Te, $\mathrm{Zn}, \mathrm{Sn}$, and Tl (Fig. 3 and table S7), known to be sensitive to open-system heating and volatilization, indicate that most of SM avoided metamorphic (on a million-year time scale) heating above $400^{\circ}$ to $500^{\circ} \mathrm{C}(29)$. Like other $\mathrm{CM}$ chondrites, both lithologies of SM51 (Fig. 2B) and some of those in SM2 contain abundant carbonate grains and complete chondrule pseudomorphs embedded in a phyllosilicate-rich matrix (Fig. 2, B and C). Sample SM2-5 contains clasts of incompletely altered CM material, whose matrix consists largely of submicrometer-sized olivine pseudomorphs after phyllosilicates and troilite (Fig. 2D and fig. S14). This and the complete lack of carbonates and tochilinite indicate that this particular clast has experienced thermal metamorphism to $\sim 500^{\circ} \mathrm{C}$; all other metamorphosed CMs are finds (30).

Thermoluminescence (TL) measurements (31) of SM2-1d (fig. S35) show heating to $300^{\circ} \pm 20^{\circ} \mathrm{C}$ within the last $0.2 \mathrm{My}$ (fig. S36), and it has induced TL similar to low-metamorphic-grade $\mathrm{CO}$ and $\mathrm{CV}$ chondrites and unlike other $\mathrm{CM}$ chondrites. Raman spectra of macromolecular carbon (fig. S33) suggest that SM2-9 experienced only $153^{\circ} \pm 27^{\circ} \mathrm{C}$, whereas a sample of SM12 experienced $268^{\circ} \pm 42^{\circ} \mathrm{C}$ on a million-year time scale [using the method in (32)]. In a Raman G-band center-versus-width diagram (fig. S33), SM2-9 plots between CM2 and $\mathrm{CO} 3$ chondrites, whereas SM12 trends closer to polycrystalline $\mathrm{C}$ observed in CV3 chondrites.

There are various potential sources for the observed heating. The fast entry would have heated the meteorite surface to $>700^{\circ} \mathrm{C}$ for up to $1.5 \mathrm{~s}$, but it is unclear that this would offer enough time to alter the meteorite deeper inside. At $0.47 \mathrm{AU}$ from the Sun, small tumbling asteroids warm up to $\sim 200^{\circ} \mathrm{C}$, more at the surface, less inside. Alternatively, the recent heating could have been related to the impact that liberated the meteorite from the asteroid surface $\geq 0.05 \mathrm{Ma}$. Annealing

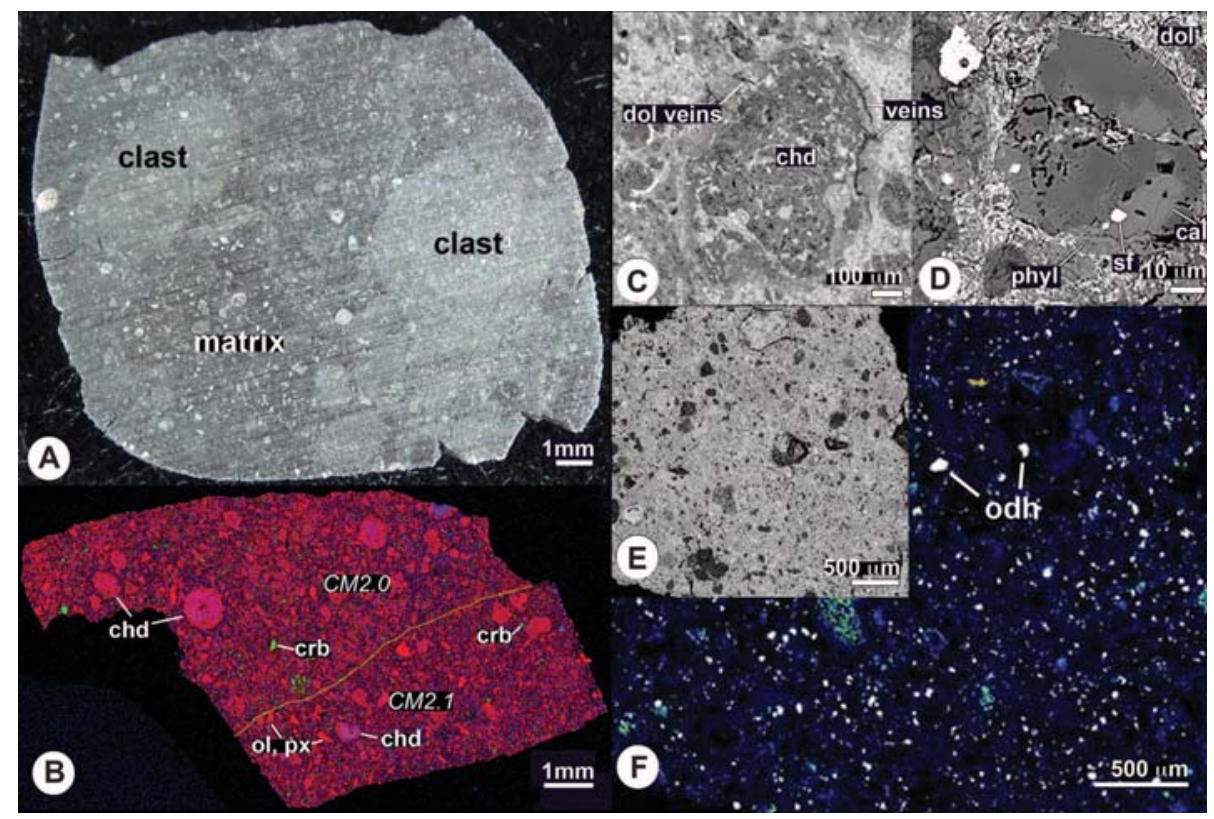

Fig. 2. (A) Slice of SM48 in visible light, showing light clasts in a dark matrix. (B) Combined elemental map in $\mathrm{Mg}$ (red), Ca (green), and Al (blue) Ka x-rays of the sample SM51-1 composed of two extensively aqueously altered CM2.0 and CM2.1 lithologies, with a sharp boundary (yellow dashed line). Both lithologies contain complete chondrule pseudomorphs (chd) embedded in a phyllosilicate-rich matrix and abundant carbonate grains (crb). In addition, the CM2.1 lithology contains rare olivine (ol) and pyroxene (px) grains of incompletely hydrated chondrules, amoeboid olivine aggregates (AOAs), and Ca-Al-rich inclusions (CAls). (C) Backscattered electron (BSE) image of a chondrule pseudomorph (chd) composed mainly of phyllosilicates in SM51-1. The fine-grained rim around the chondrule is crosscut by veins of dolomite (dol), indicating in situ aqueous alteration. (D) BSE image of a carbonate grain composed of closely intergrown dolomite (dol) and calcite (cal) with inclusions of Fe,Ni-sulfide (sf) in SM51-1. The grain is rimmed by phyllosilicates (phyl). (E) BSE image of a small chip from sample SM2-5 with (F) corresponding Ca-S composite $\mathrm{x}$-ray map (Ca, blue; $\mathrm{S}$, green); all light spots in the map correspond to oldhamite (CaS; odh). 
the decomposition of carbonates. The maximum in $\delta^{13} \mathrm{C}$ of $+65 \%$ is likely to be from the decomposition of calcite; $\delta^{13} \mathrm{C}$, however, may be underestimated because of contamination by isotopically lighter components. About $2 \%$ of the total $\mathrm{C}$ combusts above $1000^{\circ} \mathrm{C}$ and reaches a $\delta^{13} \mathrm{C}$ of $+130 \%$. This is a well-known feature of carbonaceous chondrites, produced by the combustion of ${ }^{13} \mathrm{C}$-enriched $\left(\delta^{13} \mathrm{C} \sim+1400 \%\right.$ ) presolar silicon carbide grains (35). Apparently, SM contains a significant abundance of presolar grains.
Reactive compounds. The rapid pre-rain recovery of SM offers a rare glimpse of what reactive minerals and organic compounds may be present at the surface of asteroids. Unseen in other CM chondrites, abundant $\mathrm{CaS}$ grains were found in $\mathrm{SM} 2-4$ (Fig. 2E). A powder Laue pattern of these CaS grains proved to index as oldhamite (fig. S16). Oldhamite is quickly lost to moisture. The oldhamite grains are set within fine-grained comminuted matrix, containing also olivine, enstatite, Fe-Ni-Zn sulfides, Fe-Ni$\mathrm{Cr}$ phosphides, and grains of reduced $\mathrm{C}$, suggesting

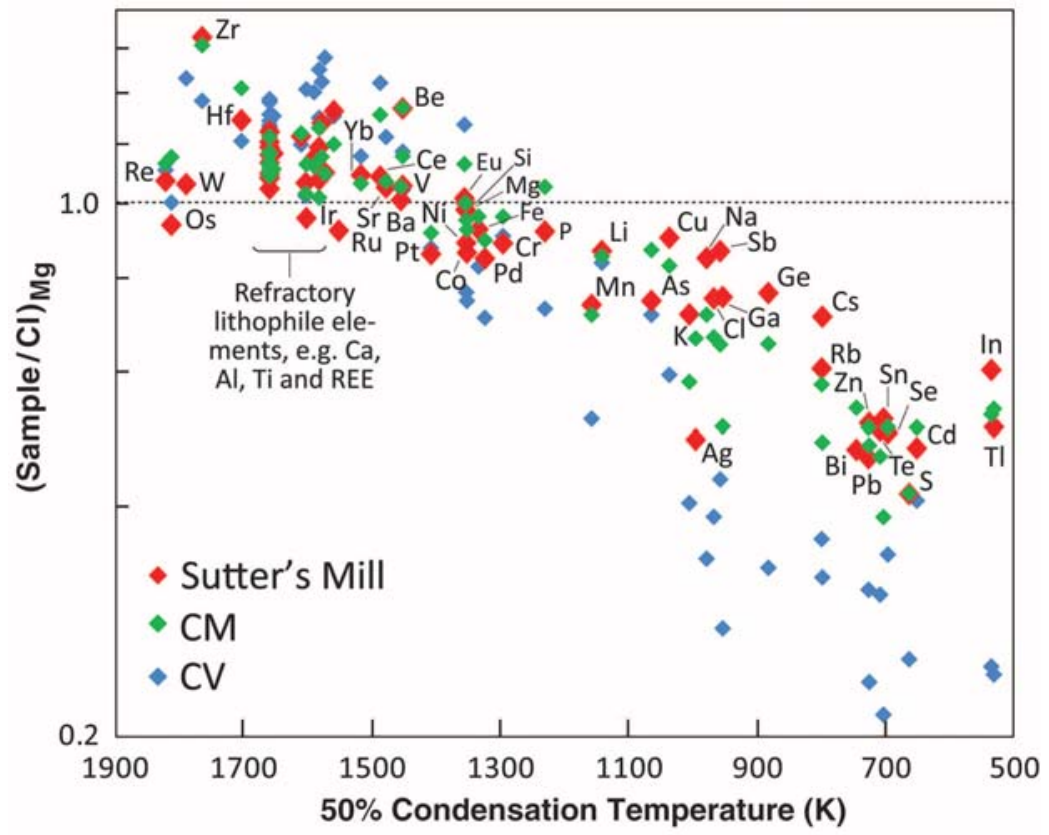

Fig. 3. Average elemental composition of SM meteorite (table S7) compared to averages for Cl (Ivuna type), $\mathrm{CM}$, and CV (Vigarano type) groups of carbonaceous chondrites. Data are normalized to $\mathrm{Cl}$ and $\mathrm{Mg}$ and plotted against 50\% condensation temperatures of the elements (41). Data sources: Cl (41), CV (42), and CM (43).

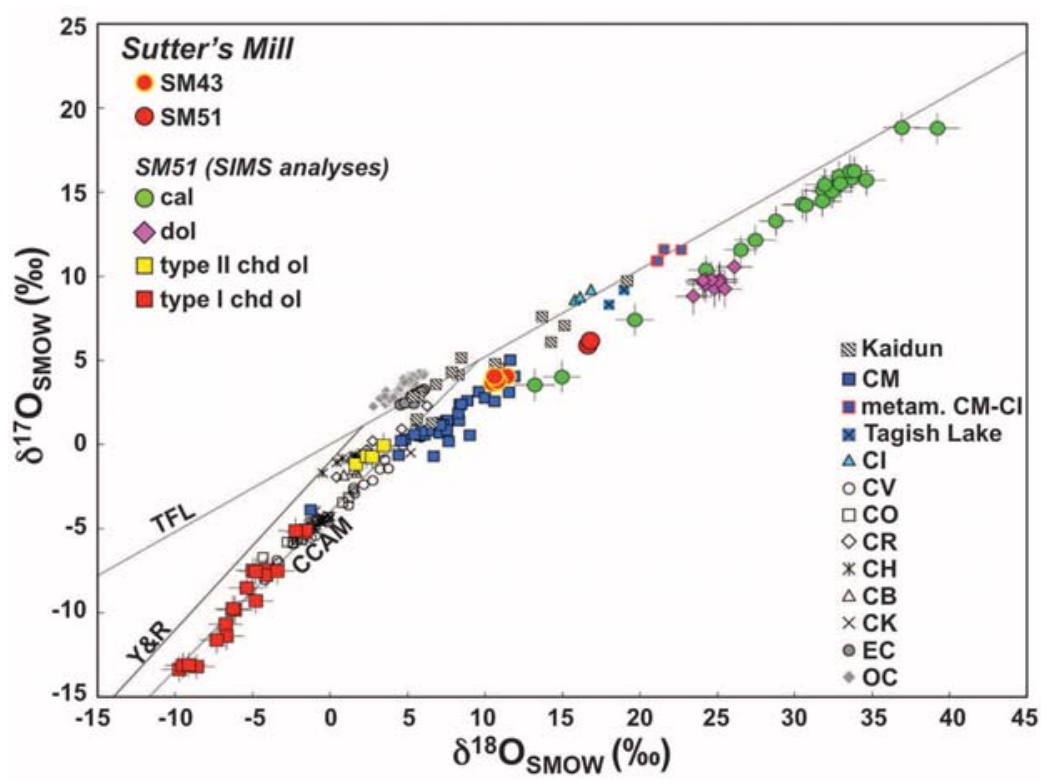

Fig. 4. SM43 and SM51 extend the currently known CM field in the three 0-isotope diagram. Data for ordinary, enstatite, and carbonaceous chondrites and for Kaidun chondritic regolith breccia are from (43-47). TFL, terrestrial fractionation line; CCAM, carbonaceous chondrite anhydrous mineral line (44); Y\&R, Young and Russell line (48). SMOW, standard mean ocean water.

admixture of a reduced component, possibly xenolithic enstatite chondrite material. It is interesting that the comminuted matrix of SM contains fragments of such a rare meteorite type, rather than the far more abundant ordinary chondrites, probably implying interactions between $\mathrm{C}$ - and E-type asteroids.

In comparison to SM2 (pre-rain), the SM12 sample (post-rain) shows strong alteration effects, demonstrating rapid reaction between terrestrial water and reactive S-bearing species. Fourier transform ion cyclotron resonance mass spectra (FTICR-MS) (36) of SM's methanol soluble fraction showed only hundreds of mass signals with comparatively low intensity, high aliphaticity, and, different from the thousands analyzed in other carbonaceous chondrites, many highly oxygenated species and polysulfur-rich compounds (fig. S31). Nuclear magnetic resonance (NMR) spectroscopy of the same extracts showed abundant highly branched, singly oxygenated aliphatics and a considerable diversity of unsaturated compounds in an intensity ratio of near 40:6:1 (fig. S32). Both NMR and FTICR-MS signals confirmed convergence with structures recently observed in rather highly thermally altered meteorites.

Water-soluble organic compounds and inorganic salts can be formed, mobilized, and altered by aqueous alteration and terrestrial weathering. Ion chromatography (37) of $\leq 20 \mathrm{mg}$ of waterextracted pre-rain SM2 showed formate at 80 parts per million (ppm) and acetate at $700 \mathrm{ppm}$, whereas post-rain interior SM12 had trace formate and only $\sim 100 \mathrm{ppm}$ acetate. Detected inorganic anions in SM2 were sulfate $(1300 \mathrm{ppm})$ and chloride (262 ppm), again with only trace amounts present in interior SM12. Murchison (presumably more aqueously altered) has a 20 times higher soluble sulfate abundance $(26,000 \mathrm{ppm})$ (37) than SM2. Sodium was the dominant cation (on a per-mole basis) at $1900 \mathrm{ppm}$ in SM2, followed by calcium (2080 ppm) and magnesium (117 ppm).

Water extracts of SM2 were analyzed by gas chromatography mass spectrometry for ammonia and amines; amino, hydroxyl, and dicarboxylic acids (38); and, for amino acids, also by liquid chromatography with fluorimetric detection and time-offlight mass spectrometry (39). The most abundant water-soluble compounds detected by these methods included glycine, $\beta$-alanine, $\gamma$-amino- $n$-butyric acid, and, in some analyses only, $\varepsilon$-amino- $n$-caproic acid (table S21). The highest total amino acid abundances were found in a fragment of SM12 at a depth of 9 to $12 \mathrm{~mm}$ from the crust. Exterior portions of SM2 and SM12 contained predominantly contaminant L-amino acids, and both samples were highly depleted in amino acids overall. The $\mathrm{pH}$ values of SM water extracts ranged from 8.90 to 9.65, which are higher than for other CMs.

Dichloromethane/methanol (9:1 volume to volume) extracts also showed variably low amounts of soluble hydrocarbon (fig. S34). SM2 contained naphthalene, methylnaphthalenes, dimethylnaphthalenes, anthracene/phenanthrene, a series of linear 15-C to 22-C alkanes, and very little $\mathrm{S}$, usually abundant in comparable CM extracts (40). The SM12 extracts were instead dominated by cyclic 
octatomic S, dimethyltrisulfide, and dimethyltetrasulfide, the latter two for about a combined $1 \mathrm{mmole} / \mathrm{g}$; the fragment contained in addition only naphthalene at 2 to $8 \mathrm{nmol} / \mathrm{g}$, plus methylnaphthalenes and biphenol in subnanomole amounts, but no alkanes or anthracene/phenanthrene.

The SM meteorite demonstrates that the complexity of C-class asteroid surfaces is greater than previously assumed. Rapid terrestrial alteration probably erases many vestiges of the internal and external processes on the asteroid that remain to be explored in spacecraft sample-return missions.

\section{References and Notes}

1. M. Fries, ]. Fries, Meteorit. Planet. Sci. 45, 1476 (2010).

2. P. Jenniskens, M. Zolensky, Meteorit. Bull. 46, 1 (2012).

3. Materials and methods are available as supplementary materials on Science Online.

4. D. R. Christie, P. Campus, in Infrasound Monitoring for Atmospheric Studies, A. Le Pichon, E. Blanc, A. Hauchecorne, Eds. (Springer, Dordrecht, Netherlands, 2010), pp. 29-75.

5. T. A. Ens, P. G. Brown, W. N. Edwards, E. A. Silber, J. Atmos. Sol. Terr. Phys. 80, 208 (2012)

6. E. A. Silber, A. Le Pichon, P. Brown, Geophys. Res. Lett. 38, L12201 (2011)

7. M. H. Shaddad et al., Meteorit. Planet. Sci. 45, 1557 (2010).

8. K. D. Smith et al., Science 305, 1277 (2004).

9. H. Haack et al., Meteorit. Planet. Sci. 47, 30 (2012)

10. H. Haack et al., in Workshop on the First Solids in the Solar System, 7 to 9 November 2011, Koloa, Kauai, Hawai'i (Lunar and Planetary Institute, Houston, TX, 2011), abstr. 9100.

11. K. Nishiizumi, M. W. Caffee, Meteorit. Planet. Sci. 44 (suppl.), 5358 (2009)

12. U. Ott, Rev. Mineral. Geochem. 47, 71 (2002).

13. V. S. Heber et al., Geochim. Cosmochim. Acta 73, 7414 (2009)

14. I. Leya, H.-]. Lange, S. Neumann, R. Wieler, R. Michel, Meteorit. Planet. Sci. 35, 259 (2000).

15. M. Gounelle et al., in The Solar System Beyond Neptune, M. A. Barucci, H. Boehnhardt, D. P. Cruikshank, A. Morbidelli, Eds. (Univ. of Arizona Press, Tucson, AZ, 2008), pp. 525-541.

16. D. Nesvorný et al., Astrophys. J. 713, 816 (2010).
17. K. J. Walsh, M. Delbo, W. F. Bottke, paper presented at the 44th American Astronomical Society/Division of Planetary Sciences Meeting, Reno, NV, 14 to 19 October, 2012, abstr. 305.04

18. A. Tsuchiyama et al., Meteorit. Planet. Sci. 44 (suppl.), 5189 (2009).

19. O. Popova et al., Meteorit. Planet. Sci. 46, 1525 (2011)

20. D. S. Ebel, M. L. Rivers, Meteorit. Planet. Sci. 42, 1627 (2007).

21. R. J. Macke, D. T. Britt, G. J. Consolmagno, Meteorit. Planet. Sci. 46, 311 (2011)

22. D. T. Britt et al., paper presented at the Meteoritical Society Meeting, Cairns, Australia, 12 to 17 August 2012, abstr. 5250.

23. M. S. Spergel, R. C. Reedy, O. W. Lazareth, P. W. Levy, L. A. Slatest, J. Geophys. Res. 91, 483 (1986).

24. P. Rochette et al., Meteorit. Planet. Sci. 43, 959 (2008).

25. ]. Gattacceca, P. Rochette, Earth Planet. Sci. Lett. 227 377 (2004).

26. A. Bischoff, E. R. D. Scott, K. Metzler, C. A. Goodrich, in Meteorites and the Early Solar System II, D. S. Lauretta, H. Y. McSween Jr., Eds. (Univ. of Arizona Press, Tucson, AZ, 2006), pp. 679-712.

27. R. J. Walker et al., Geochim. Cosmochim. Acta 66, 4187 (2002).

28. Q-Z. Yin, K. Yamashita, A. Yamakawa, R. Tanaka, B. Jacobsen, D. S. Ebel, I. D. Hutcheon, and E. Nakamura, Lunar Planet. Sci. XL, abstr. 2006 (2009)

29. ]. M. Friedrich, M.-S. Wang, M. E. Lipschutz, Meteorit Planet. Sci. 37, 677 (2002)

30. E. Tonui, M. E. Zolensky, M. E. Lipschutz, Proc. NIPR Symp. Antarct. Meteorites 15, 38 (2002).

31. M. Haq, F. A. Hasan, D. W. G. Sears, C. B. Moore, C. F. Lewis, Geochim. Cosmochim. Acta 53, 1435 (1989).

32. G. D. Cody et al., Earth Planet. Sci. Lett. 272, 446 (2008)

33. E. D. Young, R. D. Ash, P. England, D. Rumble 3rd, Science 286, 1331 (1999).

34. G. K. Benedix, L. A. Leshin, J. Farquhar, T. Jackson, M. H. Thiemens, Geochim. Cosmochim. Acta 67, 1577 (2003).

35. M. M. Grady, Astron. Geophys. 50, 4.21 (2009).

36. P. Schmitt-Kopplin et al., Proc. Natl. Acad. Sci. U.S.A 107, 2763 (2010).

37. G. W. Cooper, W. M. Onwo, ]. R. Cronin, Geochim. Cosmochim. Acta 56, 4109 (1992)

38. A. A. Monroe, S. Pizzarello, Geochim. Cosmochim. Acta 75, 7585 (2011).

39. D. P. Glavin, M. P. Callahan, J. P. Dworkin, J. E. Elsila, Meteorit. Planet. Sci. 45, 1948 (2010)

40. J. R. Cronin, S. Pizzarello, Geochim. Cosmochim. Acta 54 2859 (1990).
41. K. Lodders, Astrophys. J. 591, 1220 (2003).

42. E. Jarosewich, R. S. Clarke Jr., ]. N. Barrows, Eds., Smithson. Contrib. Earth Sci. 27, 1 (1986).

43. P. G. Brown et al., Science 290, 320 (2000)

44. R. N. Clayton, T. K. Mayeda, Geochim. Cosmochim. Acta 63, 2089 (1999).

45. R. N. Clayton, T. K. Mayeda, ]. N. Goswami, E. ]. Olsen, Geochim. Cosmochim. Acta 55, 2317 (1991).

46. R. N. Clayton, T. K. Mayeda, A. E. Rubin, J. Geophys. Res. 89, C245 (1984)

47. G. J. MacPherson et al., Geochim. Cosmochim. Acta 73, 5493 (2009).

48. E. D. Young, S. S. Russell, Science 282, 452 (1998)

Acknowledgments: The rapid recovery of SM was made possible by the local communities of Lotus, Coloma, Pilot Hill, and Grass Valley, due in part to the support of property owners, community leaders, SETI Institute and University of Caifornia at Davis (UC Davis) student and volunteer searchers, and officials of the Marshall Gold Discovery State Historic Park and the State of California. We thank J. Howard, M. Carter, D. E. Macon, and L. Hofland of the NASA Ames Research Center; M. Hill of the American Museum of Natural History; and G. Baxter, N. Botto, D. Rowland, O. Kreylos, L. Kellogg, and D. Summer of UC Davis for assistance with reported measurements. The NASA Ames Research Center-coordinated search efforts were supported by the NASA Lunar Science Institute. E.D.Y., M.Z., A.M.D., D.S.E., J.E.E., K.N., K.C.W., M.H.T., M.W.C.,

Q.Z.Y., and S.B.S. acknowledge the NASA Cosmochemistry Program for support of work reported here. In addition, the following NASA programs are sincerely acknowledged: Near Earth Object Observation Program (P.J.), Origins of Solar Systems (S.A.S.), Planetary Major Equipment (Q.-Z.Y.), Sample Return Laboratory Instruments and Data Analysis (M.W.C.), the NASA Astrobiology Institute (J.E.E., J.P.D., and D.P.G.), the SPring-8 Grant Program (M.Z.), and Post Doctoral Programs (A.S.B.). P.R.H. acknowledges support from the Tawani Foundation. R.O. acknowledges KAKENHI grants from the Japan Society for the Promotion of Science.

Supplementary Materials

www.sciencemag.org/cgi/content/full/338/6114/1583/DC1

Materials and Methods

Supplementary Text

Figs. S1 to S36

Tables S1 to S22

References (49-105)

9 July 2012; accepted 12 November 2012

$10.1126 /$ science. 1227163

\section{The Evolutionary Landscape of Alternative Splicing in Vertebrate Species}

Nuno L. Barbosa-Morais, ${ }^{1,2}$ Manuel Irimia, ${ }^{1 *}$ Qun Pan, ${ }^{1_{*}}$ Hui Y. Xiong, ${ }^{3 *}$ Serge Gueroussov, ${ }^{1,4 *}$ Leo ]. Lee, ${ }^{3}$ Valentina Slobodeniuc, ${ }^{1}$ Claudia Kutter, ${ }^{5}$ Stephen Watt ${ }^{5}$ Recep Çolak, ${ }^{1,6}$ TaeHyung Kim, ${ }^{1,7}$ Christine M. Misquitta-Ali, ${ }^{1}$ Michael D. Wilson, ${ }^{4,5,7}$ Philip M. Kim, ${ }^{1,4,6}$ Duncan T. Odom, ${ }^{5,8}$ Brendan J. Frey, ${ }^{1,3}$ Benjamin ]. Blencowe ${ }^{1,4} \dagger$

How species with similar repertoires of protein-coding genes differ so markedly at the phenotypic level is poorly understood. By comparing organ transcriptomes from vertebrate species spanning 350 million years of evolution, we observed significant differences in alternative splicing complexity between vertebrate lineages, with the highest complexity in primates. Within 6 million years, the splicing profiles of physiologically equivalent organs diverged such that they are more strongly related to the identity of a species than they are to organ type. Most vertebrate species-specific splicing patterns are cis-directed. However, a subset of pronounced splicing changes are predicted to remodel protein interactions involving trans-acting regulators. These events likely further contributed to the diversification of splicing and other transcriptomic changes that underlie phenotypic differences among vertebrate species.

$\mathrm{V}$

ertebrate species possess diverse phenotypic characteristics, yet they share similar repertoires of coding genes (1).
Evolutionary changes in transcriptomes underlie structural and regulatory differences associated with species-specific characteristics. For example, species-dependent mRNA and noncoding RNA (ncRNA) expression patterns have been linked to mutational changes in cis- and trans-acting regulatory factors, as well as to phenotypic differences (2-5). However, because organ-dependent mRNA expression levels within individual species have been largely conserved during vertebrate evolution $(6,7)$, it seems unlikely that changes in gene expression (GE)

${ }^{1}$ Banting and Best Department of Medical Research, Donnelly Centre, University of Toronto, Toronto, Ontario M5S 3E1, Canada. ${ }^{2}$ Instituto de Medicina Molecular, Faculdade de Medicina, Universidade de Lisboa, 1649-028 Lisboa, Portugal. ${ }^{3}$ Department of Electrical and Computer Engineering, University of Toronto, Toronto, Ontario M5S 3G4, Canada. ${ }^{4}$ Department of Molecular Genetics, University of Toronto, Toronto, Ontario M5S 1A8, Canada. ${ }^{5}$ University of Cambridge, CRUK-Cambridge Institute, Robinson Way, Cambridge CB2 ORE, UK. ${ }^{6}$ Department of Computer Science, University of Toronto, Toronto, Ontario M5S 2E4, Canada. ${ }^{7}$ Hospital for Sick Children, Toronto, Ontario M5G 1X8, Canada. 'Wellcome Trust Sanger Institute, Genome Campus, Hinxton, Cambridge CB10 1SA, UK.

*These authors contributed equally to this work

†To whom correspondence should be addressed. E-mail: b.blencowe@utoronto.ca 


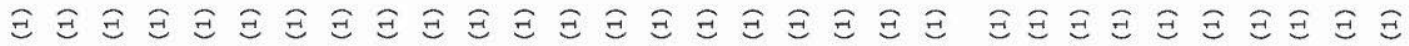

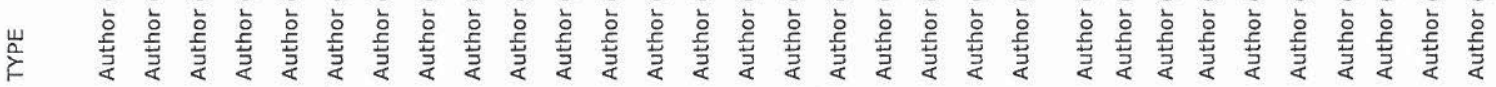

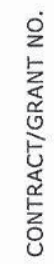

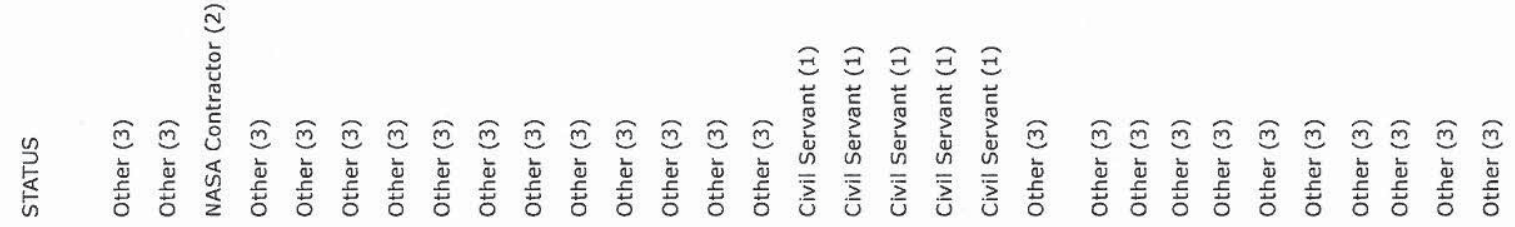

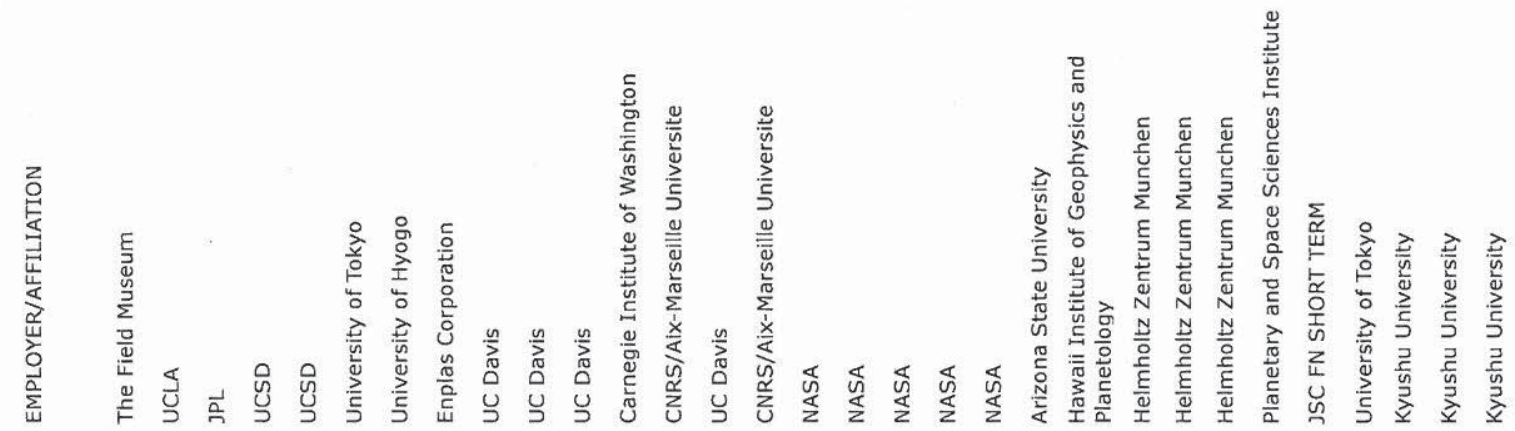

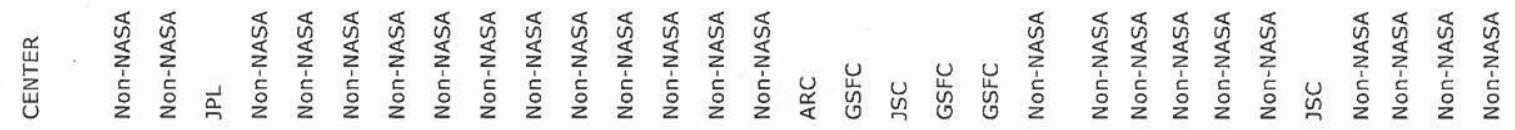

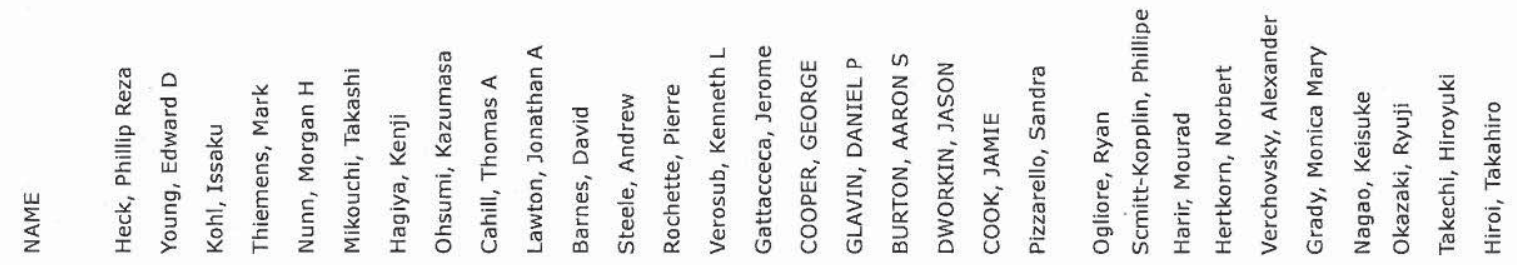

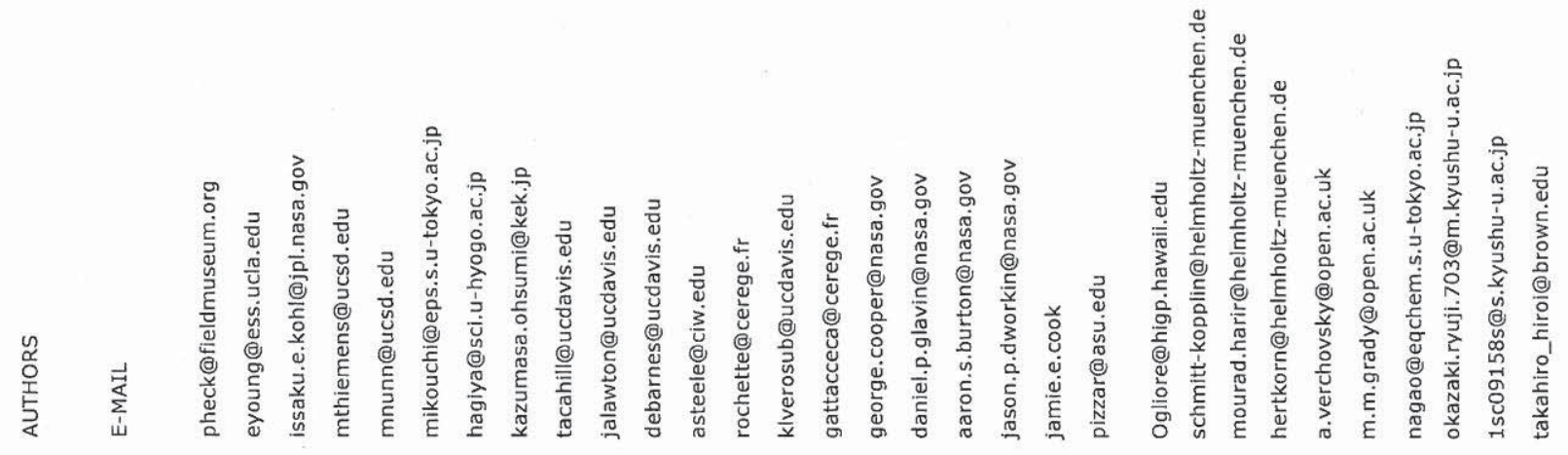




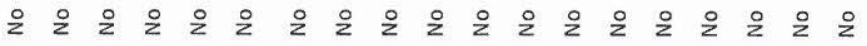

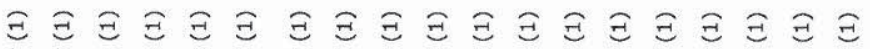

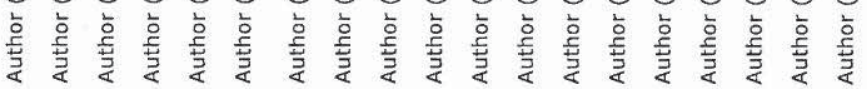

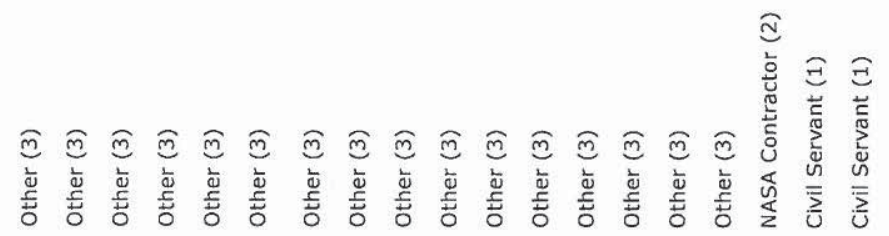

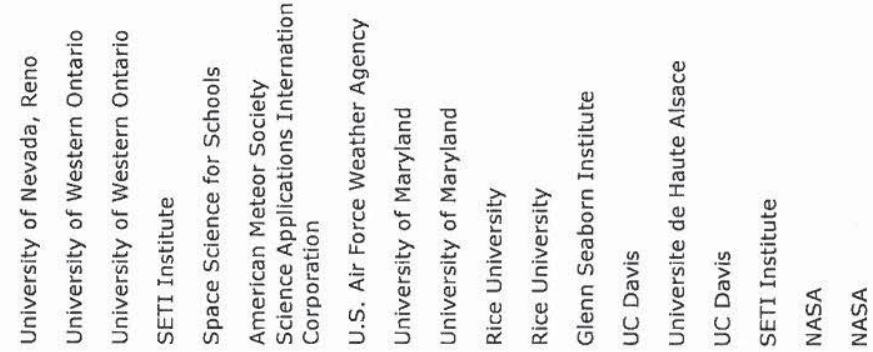

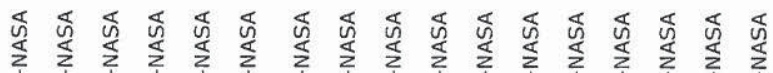

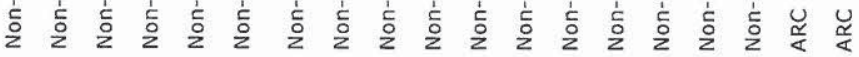

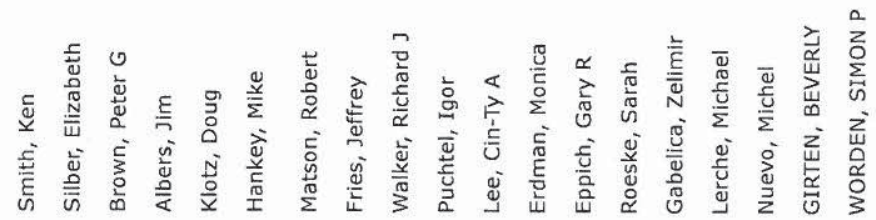

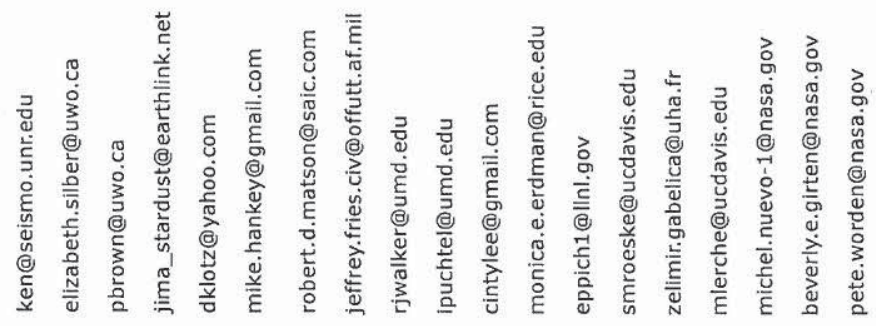

